\title{
Pathophysiological Response to Hypoxia — From the Molecular Mechanisms of Malady to Drug Discovery: Hypoxia-Inducible Factor-1 (HIF-1)-Active Cells as a Target for Cancer Therapy
}

\author{
Shinae Kizaka-Kondoh ${ }^{1, *}$, Takahiro Kuchimaru ${ }^{1}$, and Tetsuya Kadonosono ${ }^{1}$ \\ ${ }^{1}$ Department of Biomolecular Engineering, Tokyo Institute of Technology Graduate School of Bioscience and Biotechnology, \\ 4259-B60, Nagatsuta-cho, Midori-ku, Yokohama 226-8501, Japan
}

Received September 18, 2010; Accepted December 1, 2010

\begin{abstract}
The microenvironment of solid tumors is characterized by low $\mathrm{pO}_{2}$ that is well below physiological levels. Intratumoral hypoxia is a major factor contributing to cancer progression and is exacerbated as a result of oxygen consumption by rapidly proliferating tumor cells near blood vessels, poor lymphatic drainage resulting in high interstitial pressure, and irregular blood supply through immature tumor vasculature. Hypoxia-inducible factor-1 (HIF-1) is the main transcription factor that regulates cellular responses to hypoxia. Cellular changes induced by HIF-1 are extremely important targets for cancer therapy. Therefore, targeting strategies to counteract HIF-1-active cells are essential for cancer therapy. In this study, we introduce a novel strategy for targeting HIF-1-active cells.
\end{abstract}

Keywords: hypoxia-inducible factor (HIF), protein transduction domain, oxygen-dependent degradation, bioluminescence imaging, pancreatic cancer

\section{Hypoxia-inducible factors}

Hypoxia in tumors is indicated by their characteristic avascular appearance on computed tomography images and measurements of intratumoral oxygen tension (1). Hypoxia-inducible factors (HIFs) are transcription factors essential for cellular adaptation to hypoxia, and these factors regulate a wide array of genes. In cancer, both hypoxia and mutations in oncogenes and tumor-suppressor genes increase HIF activity (2). Many genes induced by HIF are critically involved in biological aspects of cancer, including immortalization, maintenance of stem cell pools, cellular differentiation, genetic instability, vascularization, metabolic reprogramming, autocrine growth factor signaling, invasion/metastasis, and resistance to treatment $(2-4)$. Therefore, early detection of HIF-active regions (intratumoral regions where HIF- $1 \alpha$ is expressed) is important to initiate timely and appropriate treatment for patients with malignant cancers.

*Corresponding author. skondoh@bio.titech.ac.jp Published online in J-STAGE on March 16, 2011 (in advance) doi: 10.1254/jphs.10R20FM
HIF-1 is the most abundant HIF, consisting of a heterodimer of HIF- $1 \alpha$ and HIF- $1 \beta$ subunits $(2,5)$. Under normoxic conditions, the HIF- $1 \alpha$ protein is degraded by ubiquitination and proteasomal degradation after hydroxylation of proline residues in the oxygen-dependent degradation (ODD) domain (6 - 9). Under hypoxic conditions, the HIF- $1 \alpha$ protein accumulates and translocates to the nucleus where it heterodimerizes with HIF-1 $\beta$ to form an active transcription factor (2). More than a hundred putative direct HIF-1 target genes have been identified, and many of these are associated with angiogenesis, invasion, and metastasis (2). Thus, HIF-1 is an excellent marker of tumor malignancy.

\section{Oxygen tension in tumor hypoxia}

The binding of pimonidazole (Pimo), which is an injectable 2-nitroimidazole derivative and a hypoxia marker, to cellular macromolecules dramatically increases below an oxygen concentration of $10 \mathrm{mmHg}$ and indicates chronic hypoxia (10). It was recently reported that HIF-1-active regions do not overlap Pimo-bound [Pimo (+)] regions (Fig. 1), but are more closely distrib- 

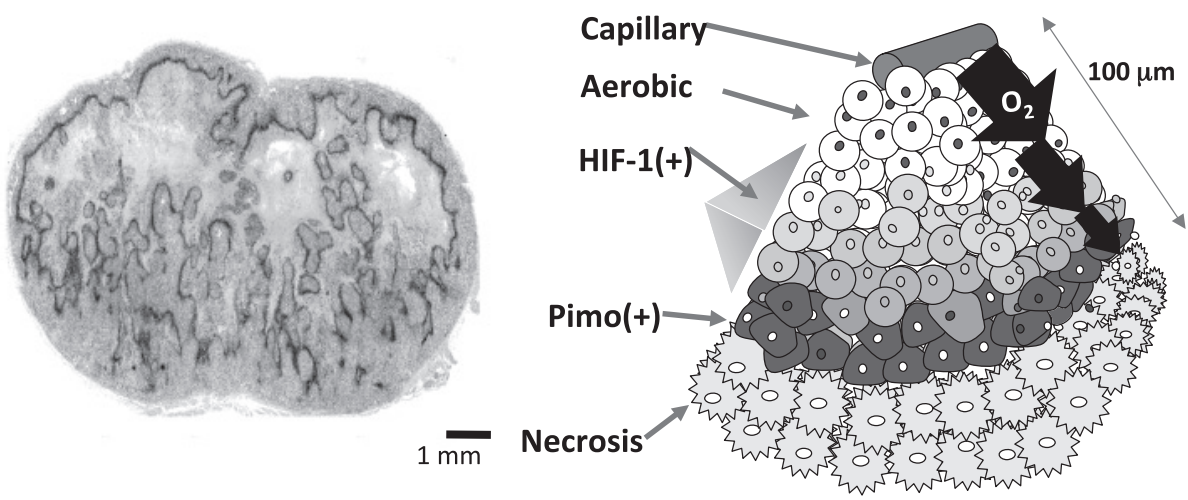

Fig. 1. Tumor hypoxia. Left, a representative tumor section after hematoxylin-eosin staining. Central areas are necrotic regions, and the surrounding darkly stained areas indicate pimonidazole $[\mathrm{Pimo}(+)]$ regions (see text). Right, a diagrammatical representation of part of a tumor cord surrounding a capillary. As the distance from the blood vessels increases, the concentration of oxygen diminishes. HIF-1-active regions are located closer to blood vessels than to the Pimo $(+)$ regions. The Pimo $(+)$ regions are located next to the necrotic regions and hardly express HIF-1 $\alpha$. Therefore, these regions have little HIF-1 activity. uted in blood vessels than the latter (11). Janssen et al. (12) extensively studied surgical specimens obtained from patients and reported that typical Pimo $(+)$ regions were observed at a distance from blood vessels with peaks around $80 \mu \mathrm{m}$, whereas HIF-1-active regions were more variable and without clear peaks. In addition, they observed that although the median values of the positive areas were similar for both markers (Pimo and HIF-1: $5.8 \%$ and $5.6 \%$, respectively), the median percentages of these regions were below 5\%.

Gene clusters, whose expression is induced by HIF-1, encode proteins that contribute greatly to the malignant alteration of a tumor. Thus, imaging and targeting of "hypoxic cells with HIF-1 activity" has gained importance. In addition, compounds such as Pimo function at absolute oxygen concentrations (below $10 \mathrm{mmHg}$ ), whereas oxygen concentrations at which HIF-1 functions differ among tissue cells. This indicates that the oxygen concentrations necessary for HIF-1 activation (biological hypoxia) differ in each tissue and cell type. Therefore, sensitive recognition of hypoxic status leading to disease development by using chemical compounds that respond to absolute oxygen concentrations is difficult. Therefore, bioprobes that respond to biological hypoxia are desirable.

\section{In vivo imaging of HIF-1-active cells}

Visualization of HIF-1 activity in vivo provides useful information about the tumor microenvironment because the presence of this activity indicates the expression of HIF-1-responsive genes that cause the aforementioned dynamic changes in the cells. Several reporter genes such as those encoding $\beta$-galactosidase $(\beta$-gal), enhanced green fluorescent protein (EGFP), and firefly luciferase were used under the control of HIF-1-dependent promoters to directly monitor HIF-1 activity in vivo and in vitro (13-17). Among the HIF-1-dependent reporter genes, luciferase reporter genes are the most suitable for quantitative monitoring of ongoing biological processes in vivo because these genes allow imaging of bioluminescence up to several centimeters in tissues with low intrinsic bioluminescence (18). Thus, bioluminescence imaging with remarkably high signal-to-noise ratios can be achieved. We established human cancer cell lines that have an integrated firefly luciferase reporter gene downstream of an artificial HIF-1-dependent promoter 5HRE (5HRE-Luc) (Fig. 2A). These cell lines express significantly higher levels of luciferase under hypoxic conditions compared with those under normoxic conditions (Fig. 2: B and C). When these cells are grafted into nude mice, the luciferase protein that responds to HIF-1-active cells is expressed and a visible light for a fixed period of time is produced after the substrate luciferin is administered. Bioluminescence imaging can be achieved using an in vivo imaging system $\left(\right.$ IVIS $^{\circledR}$; Caliper Life Sciences, Alameda, CA, USA) equipped with an ultrasensitive cooled charge-coupled device camera. Immunohistochemical analysis showed that the regions detected using anti-luciferase antibody were present at locations near the Pimo $(+)$ regions, which in turn were located at the boundary areas between viable and necrotic regions (17, 19). These results indicate that the cells expressing luciferase were certainly hypoxic. Thus, we successfully imaged HIF-1 activity in vivo $(17,19,20)$.

\section{Imaging of HIF-1-active cells after xenografting}

We monitored HIF-1 activity in human pancreatic cancer cells for several days after subcutaneously xenografting nude mice (Fig. 3: A and B). HIF-1 activity in the xenografts gradually increased for 3 days after transplantation, decreased for the next 4 days, and then increased again. These data indicate that the xenografts were exposed to a hypoxic environment for several days after transplantation, and HIF-1 activity could have 
A

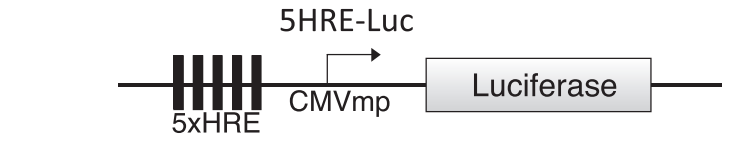

B
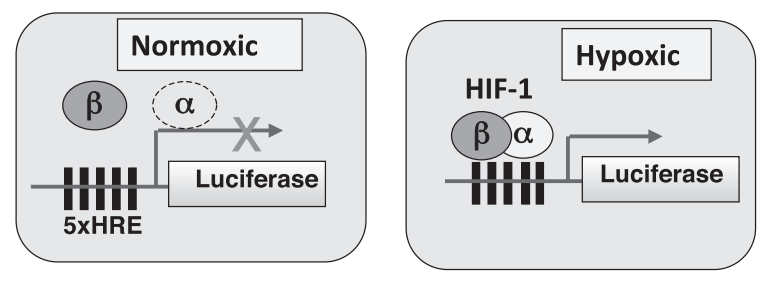

C

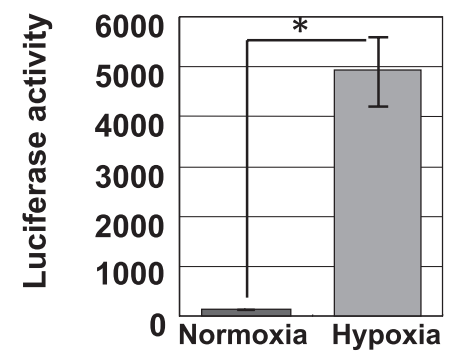

Fig. 2. Imaging of HIF-1-active cells. A: Schematic diagram of the HIF-1-dependent luciferase reporter gene 5HRE-Luc. 5HRE, 5 tandem repeats of VEGF-HRE sequences; $\mathrm{mp}$, CMV minimum promoter. B: Cancer cells carrying 5HRE-Luc express luciferase under hypoxic conditions but not under aerobic conditions. C: Quantitative analysis of luciferase activity of cancer cells with 5HRE-Luc. The cancer cells were cultured under normoxic $(21 \%)$ or hypoxic $(1 \%)$ conditions for $18 \mathrm{~h}$. Luciferase activity in the cell lysates was measured using a luminometer $(* P<0.001)$.

contributed to the angiogenesis of the xenografts, resulting in an increase in oxygen tension in the xenografts and a decrease in HIF-1 activity. These results correlated well with the ex vivo observation of xenografts with the 5HRE-EGFP reporter gene; the number of blood vessels covering the xenografts increased 5 and 6 days after transplantation, whereas EGFP fluorescence in the xenografts decreased during that period (Fig. 3C). Newly formed tumors at the metastatic site may be exposed to a similar microenvironment during their growth.

\section{Imaging of HIF-1-active cells during pancreatic cancer progression}

HIF-1-active cells are a driving force for metastasis in pancreatic cancer (20). SUIT-2/5HRE-Luc cells (a human pancreatic cancer cell line carrying 5HRE-Luc) were grafted orthotopically into the pancreas of mice. The mice who received these grafts died due to peritoneal dissemination and ascites within 7 weeks of grafting. In the absence of this in vivo imaging system, it is not pos-
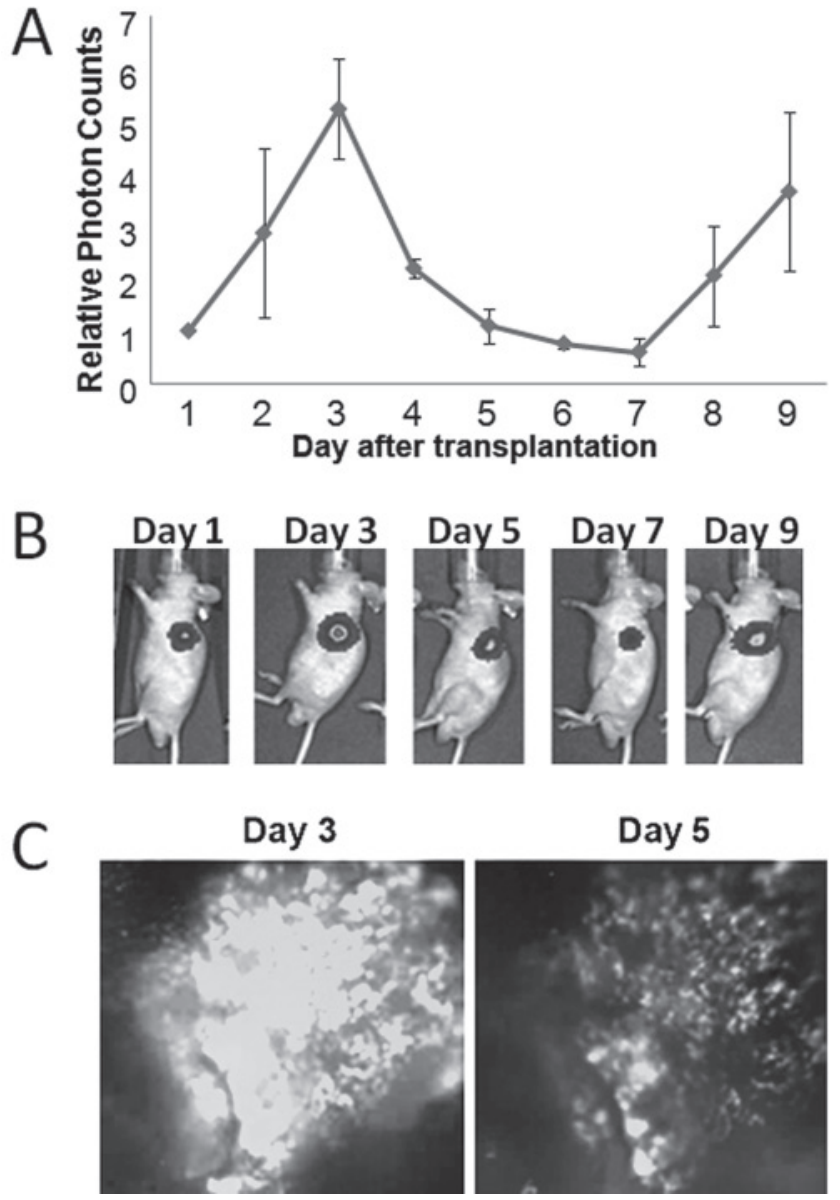

Fig. 3. Monitoring HIF-1 activity after transplantation. A: Photon counts of bioluminescence from subcutaneous tumors were measured every day after transplantation $(n=3)$. B: Representative in vivo bioluminescence images of tumors with 5HRE-Luc. The photos were obtained using IVIS ${ }^{\circledR}$ (Caliper Life Sci.). C: HIF-1 activity was visualized by green fluorescence in the tumors with the 5HRE-EGFP reporter gene. Blood vessels were visualized with AngioSense ${ }^{\mathrm{TM}} 680$ (VisEn Medical, Bedford, MA, USA). Photos were obtained using OV100 (Olympus, Tokyo). As tumor blood vessels (dark network on the tumor surface) increased at day 5 , the green fluorescent intensity (white area) indicating HIF-1 activity reduced.

sible to observe the presence of cancer until the animals are dissected. In vivo bioluminescence imaging allows for real-time noninvasive observation of proliferation, locally invaded tumors, peritoneal dissemination, and liver metastasis of HIF-1-active cancer cells (Fig. 4).

\section{Fusion proteins selectively function in HIF-1-active cells}

We previously reported that fusion with a part of the HIF-1 $\alpha$ ODD domain that contains Pro-564 residue endows the fused protein with the same oxygen-dependent regulation as HIF-1 $\alpha(21,22)$; in other words, a fusion 


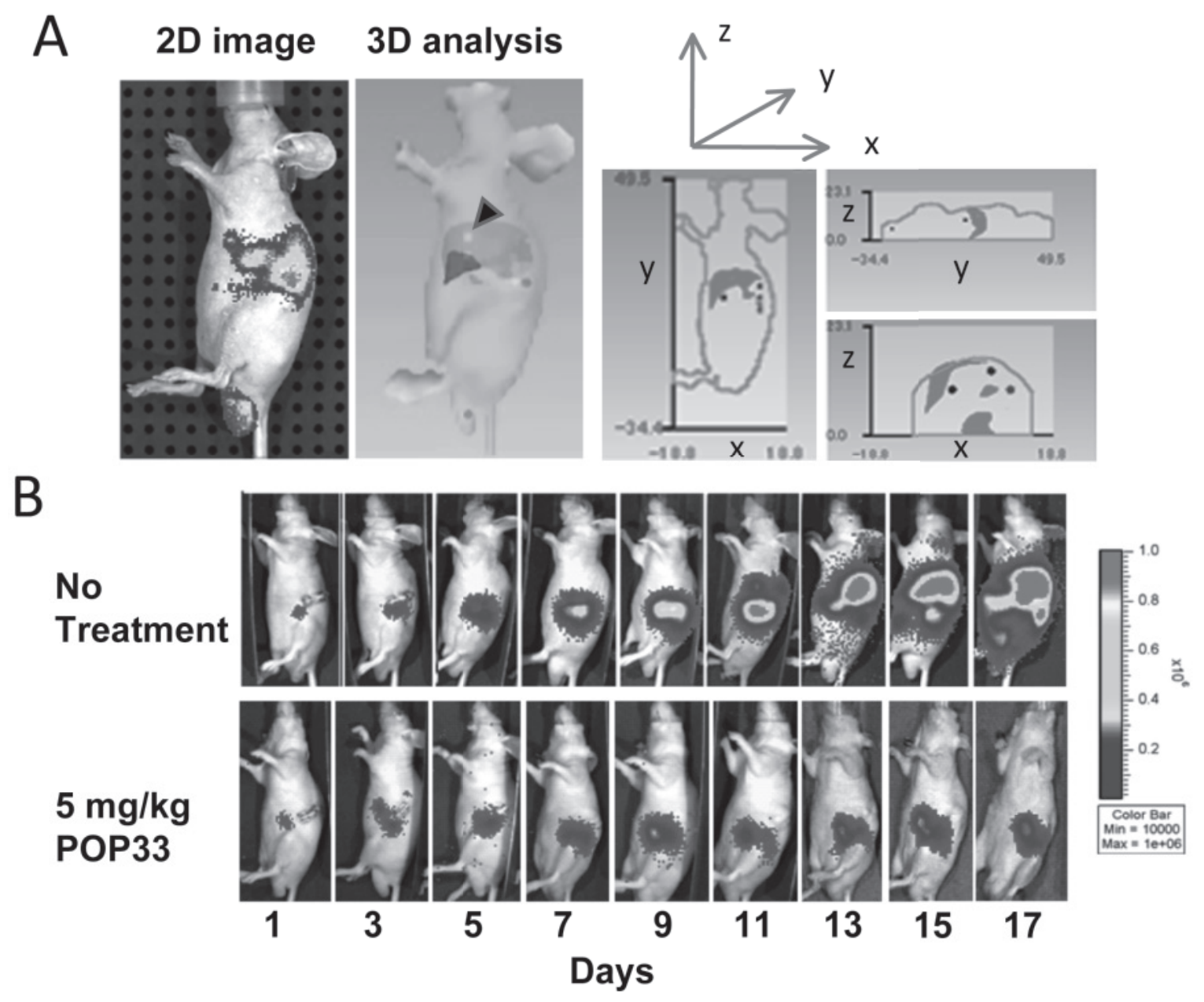

Fig. 4. Monitoring the anti-cancer effect of a fusion-protein drug specific to HIF-1-active cells. A: The human pancreatic cancer cell line SUIT-2 with 5HRE-Luc (SUIT-2 / HRE-Luc) was orthotopically transplanted into nude mice and analyzed in vivo by bioluminescence imaging. The bioluminescent signal was analyzed with 2D and 3D software (Living Image 3.10 software, Caliper Life Sciences). 2D images represent the signals detected on the body surface, while 3D images represent the signal sources in the body. The 3D images suggest liver metastasis of pancreatic cancer (arrowhead in 3D images and black dots in right panels). Right panels indicate locations of cancer cells in horizontal $(x-y)$ and vertical $(x-z, y-z)$ sections. B: Orthotopic pancreatic model mice were untreated (upper panels) or treated with POP33 [Protein-transduction domain 3 (PTD) - Oxygen-dependent degradation domain (ODD) - Procaspase-3 fusion protein] every 4th day. HIF-1 activity in the treated mice was significantly suppressed during the treatment periods.

protein with an ODD function that "stabilizes in HIF-1active cells and degrades immediately in cells without HIF-1 activity" can be created. We first determined the optimal ODD region required to impart a fused protein with the ODD function by constructing a series of ODDdomain deletion mutants and fusing them to $\beta$-gal. The optimal region with the ODD function was determined by comparing $\beta$-gal activity in the cells expressing ODD $-\beta$-gal under aerobic and hypoxic conditions. When $\mathrm{ODD}_{548-603}$ was fused to $\beta$-gal, maximum ODD regulation (hypoxia/normoxia ratio) was observed (21). Similar results were obtained with luciferase (16). ODD fusion proteins can penetrate the cell membrane because ODD regulation occurs in cells. The protein transduction domain (PTD), typically consisting of $8-20$ amino acids, contributes to the efficient entry of the fusion protein into cells and delivery of the fusion protein to the whole body (23). We fused $\mathrm{ODD}_{548-603}$ to PTD polypeptides to construct a PTD-ODD fusion protein.

The PTD-ODD ${ }_{548-603}-\beta$-gal fusion protein entered cells with almost $100 \%$ efficiency. To investigate the biodistribution and $\beta$-gal activity of the PTD-ODD $-\beta$ gal fusion protein in vivo, the protein was intraperitoneally administered to tumor-bearing mice. When PTD- $\beta$ gal was administered, protein and $\beta$-gal activities were observed in both normal hepatic and tumor tissues, 
whereas when the PTD-ODD- $\beta$-gal protein was administered, no protein or $\beta$-gal activity was observed in normal tissues and only partial activity was observed in tumor tissues (21). In brief, these findings suggest that most normal and tumor tissues are sufficiently exposed to oxygen and have no HIF-1 activity and that hypoxic HIF-1-active cells exist only in certain portions of the tumor. For further examination, we stained tumor sections with Pimo and observed $\beta$-gal protein in regions similar to Pimo $(+)$ regions. As expected, we concluded that the degradability of the PTD-ODD fusion protein is controlled in an oxygen-dependent manner, and its distribution and function have the same specificity as HIF$1 \alpha$, thus making specific imaging and targeting of HIF1-active cells feasible when the functional domain of the PTD-ODD fusion protein has both imaging and targeting functions.

\section{Development of fusion proteins that specifically target the HIF-1-active tumor microenvironment}

We developed a fusion protein, PTD-ODD ${ }_{548-603-}$ procaspase-3 $(20,21)$. The procaspase- 3 domain is an inactive proenzyme form of human caspase- 3 that is activated by pre-existing apoptotic signals in cells under hypoxic stress. PTD-ODD-procaspase- 3 has been shown to specifically eradicate HIF-1-active hypoxic cells (17, $19-22,24-26)$.

Systemic administration of PTD-ODD-procaspase-3 significantly suppressed tumor growth and reduced tumor size without any apparent side effects in mice bearing subcutaneous xenografts $(17,19)$. The hypoxic tumor cell-specific effect of PTD-ODD-procaspase-3 was confirmed in the aforementioned mouse orthotopic pancreatic cancer model (20). In this model, when HIF-1specific bioluminescence spread to the abdomen, the $\mathrm{pO}_{2}$ levels decreased significantly. When the abdominal cavity was filled with ascites and a strong signal was detected throughout the abdomen, $\mathrm{pO}_{2}$ of the abdominal cavity was less than $10 \mathrm{mmHg}(20,25) \cdot \mathrm{pO}_{2}$ of the ascites was also measured and was found to be similar to that of the abdominal cavity (20). HIF-1 activity was reciprocally correlated with oxygen tension in both the abdominal cavity and ascites. PTD-ODD-procaspase-3 significantly suppressed locally invaded tumors and metastasis of the pancreatic cancer (Fig. 4B) and improved the survival rate (20).

Our ultimate goal is to improve the effect of radiotherapy and chemotherapy on solid tumors by eradicating treatment-resistant HIF-1-active/hypoxic tumor cells. In fact, we recently reported that the combination of PTDODD-procaspase-3 and radiotherapy significantly suppresses long-term tumor growth and neovascularization
(19). This indicates that PTD-ODD-procaspase-3 targets both radiation- and hypoxia-dependent increases in HIF1, thereby suppressing HIF-1-dependent expression of survival factors such as proangiogenic and growth factors in treated tumors. We recently analyzed a wellcharacterized animal model of bone metastases in MDA-MB-231 human breast cancer cells with PTDODD-procaspase-3. The results indicated that hypoxia and HIF-1 expression contributed to the development of bone metastases (26). These results confirm that HIF1 -active cells play a crucial role in invasion and metastasis.

\section{Conclusion}

In addition to hypoxia, genetic changes in the components of the PI3K-mTOR signal pathways, in oncogene products such as ras and raf, and in tumor suppressor proteins such as von Hippel-Lindau and p53 induces increased expression of HIF $\alpha$ expression $(2,27)$. These changes and the subsequent HIF activation are associated with malignant progression. Therefore, HIF-active cells are markers not only for hypoxia but also for the malignant potential of tumor cells. Furthermore, diseases in which HIF-active cells are involved include the 3 most prevalent disorders in developed countries, namely cancer, cardiac infarction, and cerebral infarction. The prevalence of these diseases is expected to increase in the future. Therefore, novel strategies for targeting HIF-active cells are desirable for effective treatments of these diseases.

\section{References}

1 Vaupel P. Tumor microenvironmental physiology and its implications for radiation oncology. Semin Radiat Oncol. 2004;14: 198-206.

2 Semenza G. Targeting HIF-1 for cancer therapy. Nat Rev Cancer. 2003;3:721-732.

3 Brown JM, Wilson WR. Exploiting tumour hypoxia in cancer treatment. Nat Rev Cancer. 2004;4:437-447.

4 Harris AL. Hypoxia - A key regulator factor in tumor growth. Nat Rev Cancer. 2002;2:38-47.

5 Jiang B, Semenza GL, Bauer C, Marti HH. Hypoxia-inducible factor 1 levels vary exponentially over a physiologically relevant range of $\mathrm{O}_{2}$ tension. Am J Physiol. 1996;271:C1172-C1180.

6 Epstein AC, Gleadle JM, McNeil LA, Hewitson KS, O'Rourke J, Mole DR, et al. C. elegans and mammalian homologs define a family of dioxygenases that regulate HIF by prolyl hydroxylation. Cell. 2001;107:43-54.

7 Ivan M, Kondo K, Yang H, Kim W, Valiando J, Ohh M, et al. $\mathrm{HIF} \alpha$ targeted for VHL-madiated destruction by proline hydroxylation: inplicaitons for $\mathrm{O}_{2}$ sensing. Science. 2001;292:464-468.

8 Jaakkola P, Mole DR, Tian YM, Wilson MI, Gielbert J, Gaskell SJ, et al. Targeting of HIF-1 to the von Hippel-Lindau ubiquitylation complex by $\mathrm{O}_{2}$-regulated prolyl hydroxylation. Science. 
2001;292:468-472.

9 Maxwell PH, Wiesener M, Chang GW, Clifford SC, Vaux EC, Cockman ME, et al. The tumour suppressor protein VHL targets hypoxia-inducible factors for oxygen-dependent proteolysis. Nature. 1999;399:271-275.

10 Raleigh JA, Chou SC, Arteel GE, Horsman MR. Comparisons among pimonidazole binding, oxygen electrode measurements, and radiation response in $\mathrm{C} 3 \mathrm{H}$ mouse tumors. Radiat Res. 1999; 151:580-589.

11 Sobhanifar S, Aquino-Parsons C, Stanbridge EJ, Olive P. Reduced expression of hypoxia-inducible factor-1alpha in perinecrotic regions of solid tumors. Cancer Res. 2005;65:7259-7266.

12 Janssen HL, Haustermans KM, Balm AJ, Begg AC. Hypoxia in head and neck cancer: how much, how important? Head Neck. 2005;27:622-638.

13 Vordermark D, Kraft P, Katzer A, Bölling T, Willner J, Flentje M. Glucose requirement for hypoxic accumulation of hypoxiainducible factor-1alpha (HIF-1alpha). Cancer Lett. 2005;230: 122-133.

14 Liu J, Qu R, Ogura M, Shibata T, Harada H, Hiraoka M. Realtime imaging of hypoxia-inducible factor-1 activity in tumor xenografts. J Radiat Res. 2005;46:93-102.

15 Cao Y, Li CY, Moeller BJ, Yu D, Zhao Y, Dreher MR, et al. Observation of incipient tumor angiogenesis that is independent of hypoxia and hypoxia inducible factor-1 activation. Cancer Res. 2005;65:5498-5505.

16 Harada H, Kizaka-Kondoh S, Itasaka S, Shibuya K, Morinibu A, Shinomiya K, et al. The combination of hypoxia-response enhancers and an oxygen-dependent proteolytic motif enables real-time imaging of absolute HIF-1 activity in tumor xenografts. Biochem Biophys Res Commun. 2007;360:791-796.

17 Harada H, Kizaka-Kondoh S, Hiraoka M. Optical imaging of tumor hypoxia and evaluation of efficacy of a hypoxia-targeting drug in living animals. Mol Imaging. 2005;4:182-193.
18 Sadikot RT, Blackwell TS. Bioluminescence imaging. Proc Am Thorac Soc. 2005;2:537-540.

19 Harada H, Kizaka-Kondoh S, Li G, Itasaka S, Shibuya K, Inoue $\mathrm{M}$, et al. Significance of HIF-1-active cells in angiogenesis and radioresistance. Oncogene. 2007;26:7508-7516.

20 Kizaka-Kondoh S, ltasaka S, Zeng L, Tanaka S, Zhao T, Takahashi Y, et al. Selective killing of hypoxia-inducible factor1 -active cell improves survival in a mouse model of invasive and metastatic pancreatic cancer. Clin Cancer Res. 2009;15:34333441.

21 Harada H, Hiraoka M, Kizaka-Kondoh S. Anti-tumor effect of TAT-ODD-Caspase-3 fusion protein specifically stabilized and activated in hypoxic tumor cells. Cancer Res. 2002;62:20132018.

22 Harada H, Kizaka-Kondoh S, Hiraoka M. Mechanism of hypoxiaspecific cytotoxicity of procaspase- 3 fused with a VHL-mediated protein destruction motif of HIF-1a containing Pro564. FEBS Lett. 2006;580:5718-5722.

23 Schwarze SR, Ho A, Vocero-Akbani A, Dowdy SF. In vivo protein transduction: delivery of a biologically active protein into the mouse. Science. 1999;285:1569-1572.

24 Kizaka-Kondoh S, Inoue M, Harada H, Hiraoka M. Tumor hypoxia: a target for selective cancer therapy. Cancer Sci. 2003;94: $1021-1028$.

25 Inoue M, Mukai M, Hamanaka Y, Tatsuta M, Hiraoka M, KizakaKondoh S. Effect of hypoxia-targeting prodrug on a rat malignant ascites model: an experimental therapeutic platform for targeting hypoxic malignant cells. Int J Oncol. 2004;25:713-720.

26 Hiraga T, Kizaka-Kondoh S, Hirota K, Hiraoka M, Yoneda T. Hypoxia and hypoxia-inducible factor-1 expression enhance osteolytic bone metastases of breast cancer. Cancer Res. 2007;67: 4157-4163.

27 Semenza GL. Hypoxia-inducible factor 1 and cancer pathogenesis. IUBMB Life. 2008;60:591-597. 